\title{
Resolving Power and Separation Yield of the Molecular Weight Fractionation Method Based on Solubility Difference: A Computer Simulation Survey
}

\author{
Kenji KAMIDE and Yukio MIYAZAKI \\ Textile Research Laboratory, Asahi Chemical Industry Co., Ltd., \\ Hacchonawate, Takatsuki, Osaka 569, Japan.
}

(Received September 20, 1979)

\begin{abstract}
New parameters such as the resolving power $R_{\mathrm{p}}$ and various separation yield $Y$ are defined to adequately represent fractionation efficiency in molecular weight fractionation by solubility difference. The effect of fractionation conditions (the initial polymer volume fraction $v_{\mathrm{p}}{ }^{0}$, the total fraction number $n_{\mathrm{t}}$ and the concentration dependence parameter $p$ of the polymer-solvent interaction parameter $\chi$ ) on the above efficiency parameters is clarified on binary mixtures consisting of two different polymer-to-solvent molar volume ratio $X_{1}$ and $X_{2}$ by using computer experiments, which are equivalent to 2089 single-phase separation experiments. A very high resolving power cannot be expected for a single step of the phase separation. The fractionation, combined with low $v_{\mathrm{p}}{ }^{0}$ and high $n_{t}$, affords a high separation yield, except for the high molecular weight component fraction separated by successive precipitation fractionation (SPF), whose $Y$ is always zero. In contrast to this, successive solutional fractionation (SSF) enables us to isolate the high molecular weight component much more effectively than the low component.

KEY WORDS Phase-Separation/Fractionation / Fractionation Efficiency /

Resolving Power / Separation Yield /
\end{abstract}

As early as 1944 , Flory $^{1}$ defined a parameter $\varepsilon$ representing the fractionation efficiency for the molecular weight fractionation based on the solubility difference by

$$
\varepsilon=\left[\frac{\mathrm{d} f_{x(2)}}{\mathrm{d} \ln X}\right]_{X_{\mathrm{p}}}=(\ln R) / 4
$$

where $f_{x(2)}=$ fraction of a given $X$-mer remaining in the polymer-rich phase, $X=$ the polymer-to-solvent molar volume ratio, $X_{\mathrm{p}}=X$ at $f_{x(2)}=0.5, R=$ the volume ratio of the polymer-lean phase to the polymer-rich phase. Equation 1 indicates that a large $R$, accordingly lower initial concentration $v_{\mathrm{p}}{ }^{0}$, is highly desirable to carry out the fractionation run with high efficiency. Later, Kawai ${ }^{2,3}$ showed from some simplified calculations that, in order to make $\varepsilon$ large, a smaller fraction size in a successive precipitational fractionation (i.e., the weight ratio of the polymer partitioned in the polymer-rich phase to that dissolved in the starting solution) $\rho_{\mathrm{p}}$, besides $v_{\mathrm{p}}{ }^{0}$, is also quite effective. On the basis of the results obtained from the systematic computer simulations,
Kamide and Nakayama ${ }^{4}(\mathrm{KN})$ concluded that phase separation with a large $R$ does not always furnish the fractions of narrow molecular weight distribution (MWD) and in the case of successive precipitational fractionation (SPF), which was discussed and employed exclusively in the past, a decrease in $\rho_{\mathrm{p}}$ unavoidably brings about an increase in the breadth of MWD in the fractions, particularly in smaller $\rho_{\mathbf{p}}$ regions. The validity of the above conclusion was then experimentally ascertained by Kamide and his coworkers. ${ }^{5-7} \mathrm{KN}$ prefered to use a parameter $\varepsilon^{\prime}$, defined by eq 2 , in place of $\varepsilon$ as a fractionation efficiency parameter,

$$
\varepsilon^{\prime}=\left[\frac{\mathrm{d} f_{x(2)}}{\mathrm{d} X}\right]_{X_{\mathrm{p}}}=\sigma / 4
$$

where $\sigma=$ the partition coefficient of $X$-mer in the two phases.

It should be kept in mind that both $\varepsilon$ and $\varepsilon^{\prime}$ are inadequate unfortunately for quantitative discussion as to the ease of separating the components with different $X$ from their mixtures by the fractionation 
method. Huggins ${ }^{8}$ defined other parameters, $\varepsilon_{(1)}$ and $\varepsilon_{(2)}$ as efficiency parameters for mixtures of equal molecules of two components with different $X$. These parameters are evidently limited in their applicability to only specific binary mixtures he studied. ${ }^{8}$

As is evident from the above discussion, the various efficiency parameters in early studies are only approximate and have a rather ambiguous character and it is necessary to employ a much more rigorous parameter, like the resolving power $R_{\mathrm{p}}$, most commonly used and defined in optics. In this paper, we focus attention on this important point, defining at the first step new efficiency parameters such as the resolving power $R_{\mathrm{p}}$ (eq 5) and the separation yield $Y$ (eq $9,11,13,14)$ and then we clarify the effect of the initial concentration $v_{\mathrm{p}}{ }^{0}$ and total number of fractions on these parameters in the fractionation by solubility difference, using the binary mixture with different $X$.

\section{RESOLVING POWER AND SEPARATION YIELD}

First, consider binary mixtures with two different $X\left(X_{1}\right.$ and $X_{2}, X_{1}<X_{2}$, the suffix indicating the component). Write $W_{1}{ }^{0}$ for the weight fraction of the component 1 and $W_{2}{ }^{0}\left(=1-W_{1}^{0}\right)$ for the weight fraction of the component 2 . In the case of equal weight mixtures, we obtain $W_{1}{ }^{0}=W_{2}{ }^{0}=0.5$.

A solution of the above binary mixtures is cooled down until the phase separation occurs. Here, the fractionating conditions are carefully chosen in such a manner that the weights of the polymers in polymer-lean and polymer-rich phases are equal. The weight fractions of components 1 and 2, dissolved in a polymer-lean phase and a polymerrich phase, to the total polymer in a starting solution are denoted as $W_{1(1)}, W_{2(1)}, W_{1(2)}$ and $W_{2(2)}$, respectively (Here, $\left.W_{1(1)}+W_{2(1)}+W_{1(2)}+W_{2(2)}=1\right)$. Thus, the relation,

$$
\sum_{i=1}^{2} W_{i(1)} / \sum_{j=1}^{2} \sum_{i=1}^{2} W_{i(j)}=\rho_{\mathrm{s}}=1 / 2=\left(1-\rho_{\mathrm{p}}\right)
$$

is the fractionation conditions. Here, $\rho_{\mathrm{s}}$ is the weight ratio of polymer remaining in a polymer-lean phase to that of the starting solution, and is equal to the fraction size in a successive solutional fractionation run. We define the resolving power $R_{\mathrm{p}}$ in the fractionation as the ratio $X_{1} /\left(X_{2}-X_{1}\right)$ of the original polymer, which, under the given conditions, provide fractions having the following heterogeneity.

$$
\frac{W_{1(1)}}{\sum_{i=1}^{2} W_{i(1)}} \geqslant 0.99 \text { or } \frac{W_{2(2)}}{\sum_{i=1}^{2} W_{i(2)}} \geqslant 0.99
$$

In other words, $R_{\mathrm{p}}$ is defined by,

$$
R_{\mathrm{p}}=X_{1} /\left(X_{2}-X_{1}\right)
$$

A large $R_{\mathrm{p}}$ value indicates that the fractionation makes possible the separating of each component from the mixture, even if the molar volume ratio of these components $X_{1}$ and $X_{2}$ are very close to each other. In eq 4 , we adopt for convenience a $99 \%$ level purity, which may be changed as necessary.

A binary mixture (the components $X_{1}$ and $X_{2}$, their weight fractions $W_{1}{ }^{0}$ and $\left.W_{2}{ }^{0}\right)$ in a solution was fractionated into $n_{\mathrm{t}}$ fractions by successive precipitational fractionation (SPF) or successive solutional fractionation (SSF). In this case, we write $W_{i(j)}^{k}$ for the weight fraction of $i$-th component dissolved in the phase $j(j=1$ for polymer-lean phase and $j=2$ for polymer-rich phase), separated at $k$-th step. Here, the following relations:

$$
\sum_{i=1}^{2} W_{i(1)}^{n_{\mathrm{t}}-1}+\sum_{k=1}^{n_{\mathrm{t}}-1} \sum_{i=1}^{2} W_{i(2)}^{k}=1 \quad \text { for SPF }
$$

and

$$
\sum_{i=1}^{2} W_{i(2)}^{n_{1}-1}+\sum_{k=1}^{n_{\mathrm{t}}-1} \sum_{i=1}^{2} W_{i(1)}^{k}=1 \quad \text { for SSF }
$$

are satisfied.

In SPF, we write $k_{1, \mathrm{p}}$ for minimum $k$, which satisfies the condition of

$$
W_{1(1)}^{k} / \sum_{i=1}^{2} W_{i(1)}^{k} \geqslant 0.99 .
$$

Then, the following relation holds

$$
\sum_{i=1}^{2} W_{i(1)}^{k_{1}, \mathrm{p}}+\sum_{k=1}^{k_{1, \mathrm{p}}} \sum_{i=1}^{2} W_{i(2)}^{k_{1}, \mathrm{p}}=1
$$

The size of the fraction at $k$-th step $\rho_{\mathrm{p}}{ }^{k}$ is given in SPF by

$$
\rho_{\mathrm{p}}{ }^{k}=\sum_{i=1}^{2} W_{i(2)}^{k} .
$$

In this paper,

$$
\rho_{\mathrm{p}}{ }^{k}=\rho_{\mathrm{p}}=1 / n_{\mathrm{t}}, \quad k=1,2,3, \cdots, n_{\mathrm{t}}-1
$$

is assumed (i.e., the fraction size $\rho_{\mathrm{p}}$ is kept constant in a give run) and in this case, the separation yield, $Y$, is defined by 


$$
\begin{gathered}
Y_{1, \mathrm{p}}=\left(n_{\mathrm{t}}-k_{1, \mathrm{p}}\right) \rho_{\mathrm{p}} / W_{1}{ }^{0}=\left(1-k_{1, \mathrm{p}} \rho_{\mathrm{p}}\right) / W_{1}{ }^{0} \\
k_{1, \mathrm{p}} \geqslant W_{2}{ }^{0} n_{\mathrm{t}}
\end{gathered}
$$

where $k_{1, \mathrm{p}}$ is equal to $W_{2}^{0} n_{\mathrm{t}}$ at $Y_{1, \mathrm{p}}=1$. Equation 9 shows that $Y_{1, p}$ is the ratio of the weight of the fractions (as polymer-lean phase) of $99 \%$ or higher purity to the total weight of component 1 polymer existing in the starting mixture.

By analogy with $Y_{1, \mathrm{p}}$, the separation yield $Y_{2, \mathrm{p}}$ of the component 2 polymer by SPF in polymer-rich phase is given by,

$$
Y_{2, \mathrm{p}}=k_{2, \mathrm{p}} \rho_{\mathrm{p}} / W_{2}^{0}
$$

where

$$
k_{2, \mathrm{p}} \leqslant W_{2}{ }^{0} \cdot n_{\mathrm{t}}
$$

$k_{2, \mathrm{p}}$ is the maximum fractionation step, satisfying the condition of

$$
W_{2(2)}^{k} / \sum_{i=1}^{2} W_{i(2)}^{k} \geqslant 0.99 .
$$

Obviously, the component 1 polymer exists mainly in the polymer-lean phase at higher fractionation steps $\left(k \geqslant k_{1, \mathrm{p}}\right)$ and the component 2 polymer is in the polymer-rich phase at lower fractionation steps $\left(k \leqslant k_{2, \mathrm{p}}\right)$.

In SSF, the separation yield of the components 1 and $2, Y_{1, \mathrm{~s}}$ and $Y_{2, \mathrm{~s}}$ are defined by eq 13 and 14 , respectively.

$$
Y_{1, \mathrm{~s}}=k_{1, \mathrm{~s}} \rho_{\mathrm{s}} / W_{1}^{0}
$$

and

$$
Y_{2, \mathrm{~s}}=\left(1-k_{2, \mathrm{~s}} \rho_{\mathrm{s}}\right) / W_{2}^{0}
$$

where

$$
\begin{aligned}
& k_{1, \mathrm{~s}} \leqslant W_{1}^{0} n_{\mathrm{t}} \\
& k_{2, \mathrm{~s}} \geqslant W_{1}^{0} n_{\mathrm{t}}
\end{aligned}
$$

$k_{1, \mathrm{~s}}$ or $k_{2, \mathrm{~s}}$ is maximum and minimum $k$, respectively, satisfying the condition of

$$
W_{1(1)}^{k} / \sum_{i=1}^{2} W_{i(1)}^{k} \geqslant 0.99
$$

or

$$
W_{2(2)}^{k} / \sum_{i=1}^{2} W_{i(2)}^{k} \geqslant 0.99
$$

and in SSF

$$
\rho_{\mathrm{s}}{ }^{k}=\rho_{\mathrm{s}}=1 / n_{\mathrm{t}}=\sum_{i=1}^{2} W_{i(1)}^{k}
$$

Polymer J., Vol. 12, No. 3, 1980
In order to separate the component 1 polymer by SSF, it is recommended to separate the binary mixture up to the $k_{1, \mathrm{~s}}$ step and then to gather the polymers obtained at each step into the polymer-lean phases (that is, further separation beyond the $k_{1, \mathrm{~s}+1}$ step is meaningless). On the other hand, in order to isolate the component 2 polymer by SSF, it is best to fractionate the mixture up to the $k_{2, \mathrm{~s}}$ step and then to take the polymer into the polymer-rich phase at the step as the component 2 and the fractions obtained at steps before $k_{2, \mathrm{~s}}$ should be discussed.

\section{COMPUTER EXPERIMENT}

The computer experiments were carried out exactly on the basis of the modified Flory-Huggins theory, in which the concentration dependence of a polymer-solvent interaction parameter $\chi$ is expressed by the relation,

$$
\chi=\chi_{0}\left(1+p v_{\mathrm{p}}\right)
$$

$\left(\chi_{0}=\right.$ parameter, $v_{\mathbf{p}}=$ polymer volume fraction, $p=$ concentration dependence parameter), and the Gibbs' phase equilibrium theory on the multicomponent system. The calculation was performed, using the method of Kamide and Sugamiya, ${ }^{9}$ by an electronic computer IBM system 370-158.

Binary mixtures with $X_{1}=30-600$ and $X_{2}=40$ $6000\left(X_{1}<X_{2}\right)$ were assumed as the starting polymeric materials.

The following two kinds of experiments were performed:

\section{Experiment 1: Phase-Separation Experiment}

A solution of $1: 1$ (by weight) mixture was cooled and phase separation occurred. The separation temperature was chosen so that the weight fractions

$$
\left(\equiv \sum_{i=1}^{2} W_{i(1)} \text { or } \sum_{i=1}^{2} W_{i(2)}\right)
$$

of the polymer remaining in the two phases would be just one half of the total polymer (i.e., $\rho_{\mathrm{p}}=\rho_{\mathrm{s}}=1 / 2$ ). Using the phase separation data obtained thus, the resolving power $R_{\mathrm{p}}$ was calculated. 


\section{Experiment 2: Fractionation Experiment}

In addition to the above-mentioned $1: 1$ mixture, unequal weight mixtures were dissolved in a solvent (initial concentration $v_{\mathrm{p}}{ }^{0}=1 \times 10^{-4} \sim v_{\mathrm{p}, \mathrm{c}}^{0} \%$, here $v_{\mathrm{p}, \mathrm{c}}^{0}$ is the upper limit of $v_{\mathrm{p}}{ }^{0}: v_{\mathrm{p}, \mathrm{c}}^{0}=1 /\left(1+X_{w}\left(X_{z}\right)^{-1 / 2}\right)$, $X_{w}$ and $X_{z}$ are the weight-average and the $z$-average degree of polymerization of the original polymer, respectively), and were fractionated by SPF and SSF into $n_{\mathrm{t}}$ fractions $\left(n_{\mathrm{t}}=10 \sim 100\right)$. The values of $Y_{1, \mathrm{p}}$, $Y_{2, \mathrm{p}}, \quad Y_{1, \mathrm{~s}}$, and $Y_{2, \mathrm{~s}}$ were evaluated from the fractionation data obtained. The fractionation experiments done in this paper correspond completely to the 2089 single-phase separation experiments. In this case, $X_{1}, X_{2}, W_{1}{ }^{0}, v_{\mathrm{p}}{ }^{0}, n_{\mathrm{t}}$, and $p$ were employed as variables.

\section{RESULTS AND DISCUSSION}

Figure 1 shows the relationships between $X_{1}$ and $v_{\mathrm{p}}{ }^{0}$, observed in the phase separation of a $1: 1$ (by weight) mixture in solvent, yielding a constant $R_{\mathrm{p}}$. Figure $1 \mathrm{a}$ ), b), and c) correspond to the case of $p=0$, 0.8 , and 1.6, respectively. It is noteworthy that for a given $R_{\mathrm{p}}$, the $X_{2}$ value can be clearly determined. With an increase in the $X$ value of the original polymer $\left(X_{1}\right.$ and $X_{2}$ ), the resolving power decreases remarkably, compared at the same $v_{\mathrm{p}}{ }^{0}$. The degree of lowering $R_{\mathrm{p}}$ is markedly influenced by the solvent nature $(p)$; a small $p$ gives a large lowering of $R_{\mathrm{p}}$. For example, in the case of $p=0$, to obtain

$$
W_{1(2)} / \sum_{i=1}^{2} W_{i(1)}=W_{2(2)} / \sum_{i=1}^{2} W_{i(2)} \geqslant 0.99
$$

by a single-phase separation step, from a solution of a binary mixture $\left(X_{1}=30, X_{2}=60, W_{1}{ }^{0}=W_{2}{ }^{0}=1 / 2\right)$, a $1.3 \times 10^{-30} \%$ solution should be prepared. This value is to low to be practical, since the concentration range, in which the practical fractionation can be relatively easily carried out, is $0.1 \sim$ several $\%$. The possible combination of $X_{1}$ and $X_{2}$, constituting a binary mixture, from which the fractions with $99 \%$ purity can be separable, is infinite in number; for illustration, when a solution, in which a given binary mixture is dissolved, of a given concentration is to be fractionated, $R_{\mathrm{p}}$ increases significantly with an increase in $p$, indicating that if a solvent with a large $p$ value is employed, high resolving power can be obtained. As is evident from Figure 1, very high resolving power cannot be expected for a single step

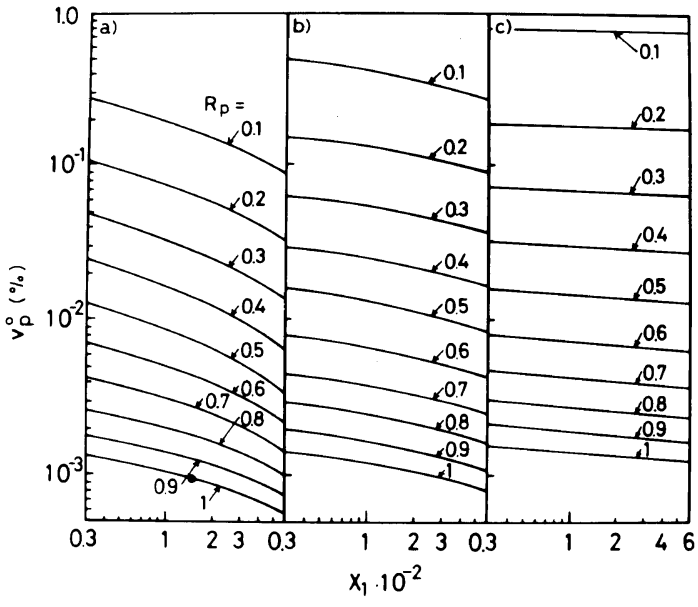

Figure 1. Effect of concentration dependence parameter $p$ on the resolving power $R_{\mathrm{p}}-X_{1}$ relations: $W_{1}{ }^{0}=W_{2}{ }^{0}=0.5$ (binary equal weight mixture); the fraction size, $\rho_{\mathrm{p}}=\rho_{\mathrm{s}}=0.5$; a) $p=0$, b) $p=0.8$, and c) $p=1.6$.

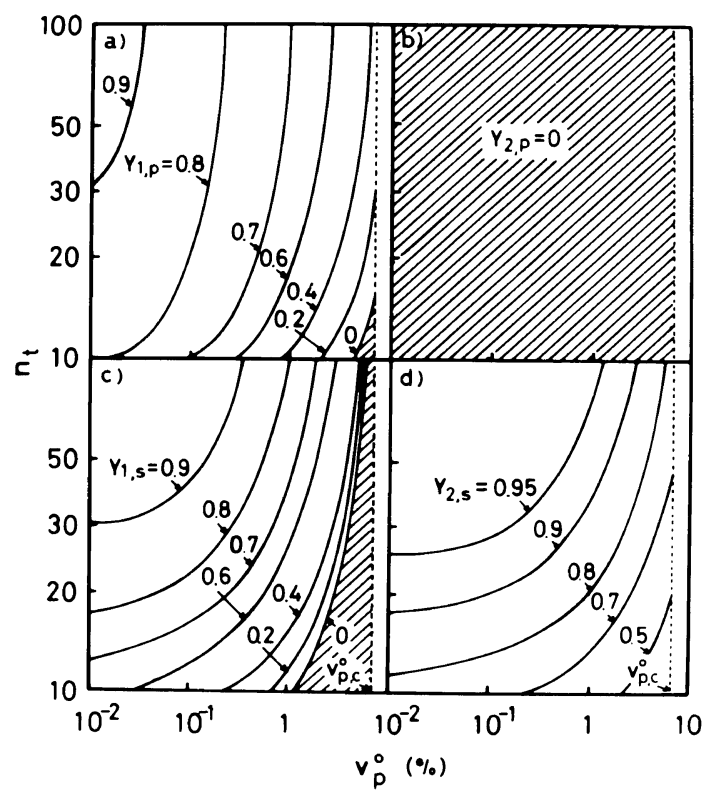

Figure 2. Relationships among initial polymer volume fraction $v_{\mathrm{p}}^{0}$, total fraction number $n_{\mathrm{t}}$, and various $Y$ : binary equal weight mixture $\left(X_{1}=150, X_{2}=300\right.$, $\left.W_{1}{ }^{0}=W_{2}^{0}=0.5\right)$; $p=0$; a) $Y_{1, \mathrm{p}}$, b) $Y_{2 \mathrm{p}}$, c) $Y_{1, \mathrm{~s}}$, and d) $Y_{2 \mathrm{~s}}$.

phase separation.

Solutions of 1 : 1 mixture $\left(X_{1}=150, X_{2}=300\right)$ with 
a concentration ranging from $10^{-2} \sim v_{\mathbf{p}, \mathrm{c}}^{0}(\equiv 6.56) \%$ was fractionated, assuming $p=0$, by SPF or SSF into $10 \sim 100$ fractions. The original mixtures are denoted as closed circles in Figure 1. Figure 2 shows the relationships between $v_{\mathrm{p}}{ }^{0}$ and $n_{\mathrm{t}}$, affording a given separation yield $Y$ calculated from the above fractionation data. Figure 2 a), b), c), and d) correspond to $Y_{1, \mathrm{p}}, Y_{2, \mathrm{p}}, Y_{1, \mathrm{~s}}$, and $Y_{2, \mathrm{~s}}$, respectively. The shadowed area in the figure is the area of zero resolving power: $Y=0$, and the dotted line means $v_{\mathrm{p}, \mathrm{c}}^{0}$. In general, to attain a given separation yield $Y$, it is necessary to separate a comparatively small number of fractions from a dilute solution or to isolate a relatively large number of fractions from solutions of high concentration. As anticipated, the fractionation, combined with low $v_{\mathrm{p}}{ }^{0}$ and high $n_{t}$, affords a high separation yield. An exception is $Y_{2, \mathrm{p}}$, which is always zero under any fractionation conditions. Thus, it should be remembered that highmolecular-weight fractions cannot be separated precisely if SPF is employed. In contrast to this, usage of SSF enables us to separate highmolecular-weight components much more effectively than low-molecular-weight components. But, in this case, the range of separation for the low $X$ component is also as wide as that for a high $X$ component. In an extremely narrow $v_{\mathrm{p}}{ }^{0}$ range, in the vicinity of $v_{\mathrm{p}, \mathrm{c}}^{0}$, the low $X$ component cannot be separated.

In order to obtain fractions having one component by weight of $99 \%$ or more, by using only one step of the phase separation, a starting concentration should be maintained as low as $9 \times 10^{-4} \%$ (see Figure 1). This is a matter of considerable difficulty. In SPF, the separation of the component 1 polymer with $99 \%$ purity is possible under not too extreme conditions (for example, $v_{\mathrm{p}}{ }^{0}=1 \%$ and $n_{\mathrm{t}}=10$ ). However, in the latter case, the separation yield $Y_{1, \mathrm{p}}$ remains about 0.4 , far smaller than $Y_{1, \mathrm{p}}=1.0$, obtained by a single-phase separation experiment. $v_{\mathrm{p}}{ }^{0}$, suitable for SPF, is almost $10^{3}$ times as large as that for a single-phase separation. Although time consuming, SPF is thus undoubtedly far more economical in comparison with a single step separation method. If SSF is employed in place of SPF, $Y_{1, \mathrm{~s}}=0$ is obtained under the conditions of $v_{\mathrm{p}}{ }^{0}=1 \%$ and $n_{\mathbf{t}}=10$. Under much more unusual conditions, for example $v_{\mathrm{p}}{ }^{0}=0.1 \%$ and $n_{\mathrm{t}}=50, \quad Y_{1, \mathrm{p}}(=0.8)<Y_{1, \mathrm{~s}}(=0.9)$ is observed, indicating that SSF affords a higher separation yield of the low component than SPF.

In this manner, under the operating conditions of a high initial concentration and small number of fractions, being apparently economically advantageous, SPF is preferable in separating the low $X$ component (i.e., the component 1 polymer) to SSF. With respect to the separation of a high $X$ component, SSF is of course more advantageous than SPF. It is of interest to note that in both SSF and SPF, the purity of the polymers remaining in a polymer-lean phase in the case of SPF or in a polymer-rich phase in SSF is always higher than that of the fractions (polymer-rich phase in SPF and polymer-lean phase in SSF).

A $1: 1$ (by weight) mixture $\left(X_{1}=150, X_{2}=300\right.$, and $W_{1}{ }^{0}=W_{2}{ }^{0}=0.5$ ) was fractionated from its $1 \%$ solution by SPF or SSF into twenty fractions $\left(n_{\mathrm{t}}=20\right)$. Figure 3 shows the effect of the $p$ value of various $Y$, calculated from the above fractionation data. In the figure, the open rectangle, closed rectangle, open circle and closed circle denote $Y_{1, \mathrm{p}}$, $Y_{2, \mathrm{p}}, Y_{1, \mathrm{~s}}$ and $Y_{2, \mathrm{~s}}$, respectively. The parameters $Y_{1, \mathrm{p}}$, $Y_{1, \mathrm{~s}}$ and $Y_{2, \mathrm{~s}}$ increase linearly with an increase in the $p$ value and these $p$ dependences are very similar for the most part. The parameter $Y_{2, \mathrm{p}}$ always remains zero, irrespective of the $p$ value employed. That is, SPF does not enable us to separate the high molecular weight component with high purity, even if the solvent is very carefully chosen.

The change in the separation yield $Y$ in SPF and $\mathrm{SSF}$ with molecular characteristics $\left(X_{1}, X_{2}\right.$, and $\left.W_{1}{ }^{0}\right)$

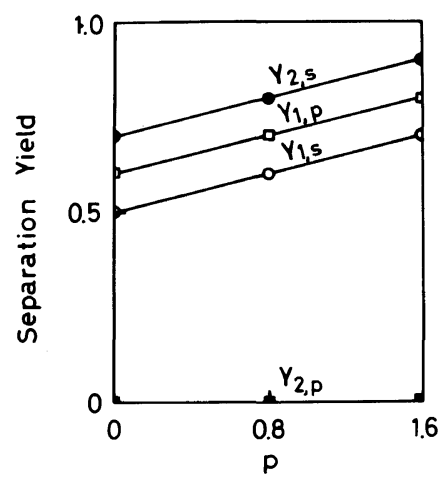

Figure 3. Effect of concentration dependence parameter $p$ on separation yield $Y$ : binary equal weight mixture $\left(X_{1}=150, X_{2}=300\right)$; initial polymer volume fraction, $v_{\mathrm{p}}{ }^{0}=1 \%$; total number of fraction, $n_{\mathrm{t}}=20 ; \square$, $Y_{1, \mathrm{p}}, \boldsymbol{\square}, Y_{2 \mathrm{p}}, \mathrm{O}, Y_{1, \mathrm{~s}}$, and $\boldsymbol{O}, Y_{2 \mathrm{~s}}$. 
of the original polymers was systematically investigated. For this purpose, the original polymers $\left(X_{1}=30 \sim 600, \quad X_{2}=40 \sim 3600, \quad W_{1}{ }^{0}=W_{2}{ }^{0}=1 / 2\right)$, dissolved in a solution of $1.0 \%$, were fractionated into 20 equal fractions. The separation yield $Y$ was calculated from the data of these computer experiments. Figure 4 shows the relation between $X_{1}$ of the original polymers and $X_{1} / \Delta X\left(=X_{2}-X_{1}\right)$, yielding constant $Y$ value. The value of $X_{1} / \Delta X$, corresponding to $Y=1$, is $R_{\mathrm{p}}$. The shadowed area in the figure is the area, in which any component with a purity of $99 \%$ or more cannot be isolated. Under these conditions, (i.e., $v_{\mathrm{p}}{ }^{0}=1 \%$ and $n_{\mathrm{t}}=20$ ), the fraction consisting of component 2 polymer only or of at least $99 \%$ purity can never be separated by SPF. Occasionally, we can not separate component 1, by SSF particularly in the case of large $X_{1} / \Delta X$. For a constant $\Delta X, Y$ decreases rapidly with an increase in $X_{1}$. To separate one component from the mixture with a given separation yield $Y$, it is necessary to keep the difference $X_{2}-X_{1}$ as large as possible for a large

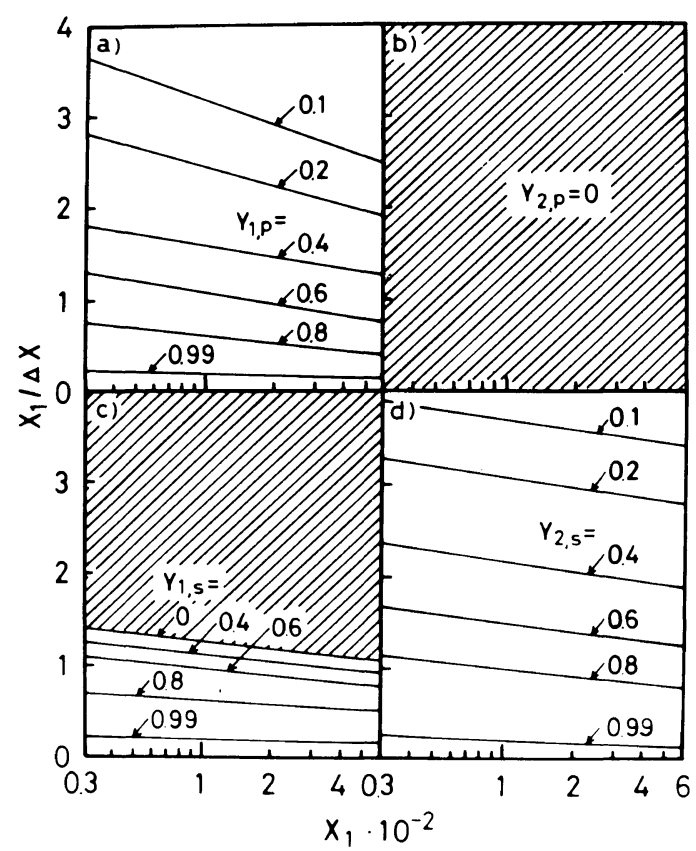

Figure 4. Relationships among resolving power $R_{\mathrm{p}}, X_{1}$, and the separation yield $Y$ : binary equal weight mixture $\left(X_{1}{ }^{0}=X_{2}{ }^{0}=0.5\right)$; initial polymer volume fraction, $v_{\mathrm{p}}{ }^{0}=1 \%$; total fraction number, $n_{\mathrm{t}}=20 ; p=0$; a) $Y_{1, \mathrm{p}}$, b) $Y_{2, \mathrm{p}}$, c) $Y_{1, \mathrm{~s}}$, and d) $Y_{2 \mathrm{~s}}$.
$X_{1}$. For instance, in order to separate the component 1 with a separation yield $Y=99 \%$ by SPF $\left(Y_{1, p}=0.99\right)$, the ratio $X_{2} / X_{1}$ should be equal to or larger than 6. Similarly, $X_{2} / X_{1} \geqslant 6$ is needed for $Y_{1, \mathrm{~s}}=0.99$. Even if the molecular characteristics of the original polymer vary widely, SPF is effective for separating the low-molecular-weight component and SSF is preferable for isolating high-molecularweight component (component 2) as found for $1: 1$ (by weight) mixtures. This is applicable for multicomponent polymers.

In the above discussion, only a $1: 1$ (by weight) mixture was taken as the original polymer. Next, the effect of the distribution of the components in the polymer on various separation yields $Y$ was studied. For this, a $1 \%$ solution of the binary mixtures $\left(W_{1}{ }^{0}=0.1 \sim 0.9, \quad X_{1}=150, \quad X_{2}=300\right) \quad$ was fractionated into equal twenty fractions $\left(n_{\mathrm{t}}=20\right)$ by using SPF or SSF. Figure 5 illustrates the relations between separation the yield $Y$ and $W_{1}{ }^{0}$, obtained by the above fractionations. $Y_{2, \mathrm{p}}=0$ was observed regardless of the composition $W_{1}{ }^{0}$. Other parameteres, except for $Y_{2, p}$, are larger if the composition of the component in question is higher (e.g., the component 1 for $Y_{1, \mathrm{~s}}$ ). This means that the predominant component can be readily isolated as a fraction with high purity and high separation yield. In the range $W_{1}^{0}<0.4, Y_{1, \mathrm{~s}}=0$ was observed. To separate the component 1 , it is strongly recommended to utilize SSF when the component 1 is predominated (i.e., $W_{1}^{0}$ is large) in the start-

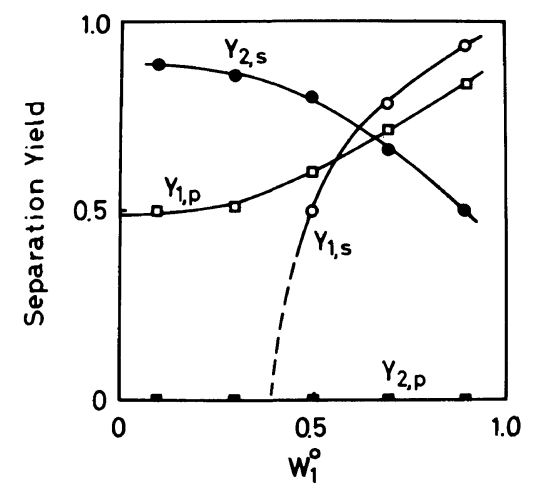

Figure 5. Effect of the composition ratio $W_{1}{ }^{0}$ of the binary mixture $\left(X_{1}=150, X_{2}=300\right)$ on various separation yields $Y$ : initial polymer volume fractions, $v_{\mathrm{p}}{ }^{0}=1 \%$; total fraction numbers, $n_{\mathrm{t}}=20 ; p=0$. 
ing polymer and to utilize SPF when $W_{1}{ }^{0}$ is small. The high-molecular-weight component can always be separated by SSF, irrespective of the composition of original polymers. With the polymer having $W_{1}^{0}>0.5$, both components can be isolated concurrently by SSF. In this sense, the use of SSF should be recommended most. This is exactly what is done in practice. In this paper the discussion is based only on the results obtained by computer experiments. Kamide et al. have confirmed the validity of the procedure of a computer simulation used here for systems such as polystyrene(PS)methylcyclohexane ${ }^{7,10}$, PS-cyclohexane $(\mathrm{CH}),{ }^{7}$ poly $\left(\alpha\right.$-methylstyrene)-mixture- $\mathrm{CH}^{11}$ by comparing the following quantities determined experimentally and theoretically: the partition coefficient $\sigma$, volume ratio of polymer-lean phase to polymer-rich phase $R$, polymer volume fraction in the two phases $v_{\mathrm{p}(1)}$ and $v_{\mathrm{p}(2)}, M_{w}$ and $M_{w} / M_{n}$ of the polymers in both phases, weight ratio of components in the fractions. Therefore, the conclusions obtained in this paper for phase-separation and fractionation of binary mixtures are likely to provide an additional important theoretical basis for molecular weight fraction of existing polymers by solubility difference.

\section{REFERENCES}

1. P. J. Flory, J. Chem. Phys., 12, 425 (1944).

2. T. Kawai, Kobunshi Kagaku, 12, 63 (1955).

3. T. Kawai, Kobunshi Kagaku, 12, 71 (1955).

4. K. Kamide and C. Nakayama, Makromol. Chem., 129, 289 (1969).

5. K. Kamide, K. Sugamiya, T. Ogawa, C. Nakayama, and N. Baba, Makromol. Chem., 135, 23 (1970).

6. K. Kamide, K. Sugamiya, T. Terakawa and T. Hara, Makromol. Chem., 156, 287 (1972).

7. K. Kamide, Y. Miyazaki and T. Abe, Makromol. Chem., 177, 485 (1976).

8. M. L. Huggins, J. Polm. Sci., A-2, 5, 1221 (1967).

9. K. Kamide and K. Sugamiya, Makromol. Chem., 139, 197 (1970).

10. K. Kamide, Y. Miyazaki, and T. Abe, Polym. J., 9, 395 (1977).

11. I. Noda, K. Kamide, Y. Miyazaki, and H. Ishikawa, Polym. Prepr., Jpn., 27, 558 (1078). 